\title{
Acoustic Impedance of Outlet of a Hard-Walled Unbaffled Cylindrical Duct for Multimode Incident Wave
}

\author{
J. JURKIEWICZ ${ }^{a}$, A. SNAKOWSKA ${ }^{b, *}$ AND D. SMOLIK ${ }^{c}$ \\ ${ }^{a}$ Faculty of Electrical Engineering, Automatics, Computer Science and Electronicsand Electronics, AGH \\ al. A. Mickiewicza 30, 30-059 Cracow, Poland \\ ${ }^{b}$ Faculty of Mechanical Engineering and Robotics, AGH, al. A. Mickiewicza 30, 30-059 Cracow, Poland \\ ${ }^{c}$ Faculty of Physics and Applied Computer Science, AGH, al. A. Mickiewicza 30, 30-059 Cracow, Poland \\ In the paper, the specific acoustic impedance of outlet of an unbaffled duct for incident wave composed of \\ one or many circumferential cut-on duct modes has been derived and presented on graphs. When considering the \\ multimode excitation the equal energy per mode principle and random phases have been assumed. Experimentally \\ verified assumption on equal energy per mode allows for quantitative analysis of the sound field and thus \\ provides more physical insight into the in-duct and out-duct phenomena. The assumption of random phase was \\ implemented by means of the Monte Carlo method and so the expected value, the standard deviation and the \\ percentile curves of the impedance were presented. Numerical data obtained according to the derived theoretical \\ formulae for single and multimode incident wave manifest strong modal character and dependence on the modes \\ phases. The assumption of multimode incident wave has brought the theoretical model closer to what is being \\ observed in practice, especially when ducts of large radius, such as heating and air conditioning systems or \\ turbojet engines, are considered.
}

PACS: $43.20 . \mathrm{Mv}, 43.20 . \mathrm{Rz}$

\section{Introduction}

Impedance is a quantity that reflects the condition of the acoustic wave propagation outside the duct and thus plays an important role in qualitative and quantitative description of the acoustic field. Before the diffraction problem was solved, the most commonly used models assumed the duct outlet located in an infinite rigid baffle. Its specific acoustic impedance for the plane wave was for the first time calculated by Rayleigh [1]. This formula is still frequently used in many technical applications, even in cases when it presents a very rough estimate. Later Zorumski [2] extended Rayleigh's results on modes other than the plane wave. Snakowska and Wyrzykowski [3] calculated the specific impedance of the unbaffled duct outlet for a single radial mode propagating towards the open end taking into account the diffraction phenomena.

The solution of the wave equation obtained for the unbaffled duct and the plane wave incident by Levine and Schwinger [4], generalized later on arbitrary single cut-on mode $[5,6]$, even though mathematically complicated, allows for deriving mathematical formula of the impedance and its numerical calculations. The results obtained within such a model describe better real-life devices containing duct-like elements. The one-mode inci-

* corresponding author; e-mail: anna.snakowska@agh.edu.pl dent wave approach is commonly applied, even by authors analyzing the sound field of modern turbofan engines [7], for which the Helmholtz number (diffraction parameter) is much above the cut-on value of many radial and circumferential modes. Anyhow, in practice the phenomena are even more complicated. Inside jet engines, but also ventilation, heating or cooling systems etc. the most frequent is the so-called multimodal excitation, when the wave heading the outlet is a superposition of many duct-modes. Mathematically it is trivial — such a wave is represented by superposition of single-mode potentials - as long as quantitative analysis, calling for knowledge of amplitudes and phases of modes constituting the incident wave, is not the goal. Thus, as Joseph and Morfey have concluded [8], comparatively little papers have been published aimed at understanding multimodal radiation, especially the phenomena at the outlet and outside the duct. The far field radiation is usually characterised by means of the root mean square pressure [8], thereby modes phases and interference effects are neglected.

In this paper, the equal energy per mode (EEpM) has been assumed, while phases have been assumed random. The model assuming equal energy per mode, presented by Snakowska [9] has became a common way of describing the multimode propagation in circular ducts [7]. However, modes phases had not been, in general, included in the field description, that was equivalent to the incoher- 
ent sources approximation.

In this paper, a strong dependence of the outlet acoustic impedance on the modes phases, based on numerical results obtained by means of the Monte Carlo method, will be examined. Assumption of multimode excitation and random phases better reflects practical acoustic fields of many duct-like devices and allows for its quantitative analysis, thus providing more physical insight into in-duct and out-duct phenomena.

\section{Governing equations}

The outlet of a semi-infinite cylindrical waveguide can be considered as a circular source located in a cylindrical baffle of given material properties, such as for example soft, hard, impedance etc. surface. Propagation of the acoustic wave inside and its radiation outside through the outlet is mathematically described by the wave equation for the acoustic pressure or velocity potential [10] together with the adequate boundary condition (Dirichlet, Neumann, impedance, etc.) given on the semi-infinite cylinder. Such mathematical formulation of the considered problem, accounting for diffraction phenomena can be solved by means of the Wiener-Hopf factorization method [11]. For harmonic excitation, which will be assumed in what follows, the wave equation reduces to the Helmholtz equation [10]. The knowledge of the acoustic pressure or potential allows for thorough qualitative and quantitative field analysis and, in particular, calculation of the acoustic impedance. For a hard/soft duct excited harmonically with a given frequency $\omega$ there is a limited number of propagating modes, depending on the duct radius $a$ [10], thus the in-duct phenomena are described by means of the diffraction parameter $k a=\omega a / c$, called also the reduced frequency or the Helmholtz number. The frequency limit between propagating and exponentially dumped modes is called the cut-off frequency. In a hard duct, the cut-off frequency of the plane wave is zero, so it propagates in a duct of any radius at any frequency.

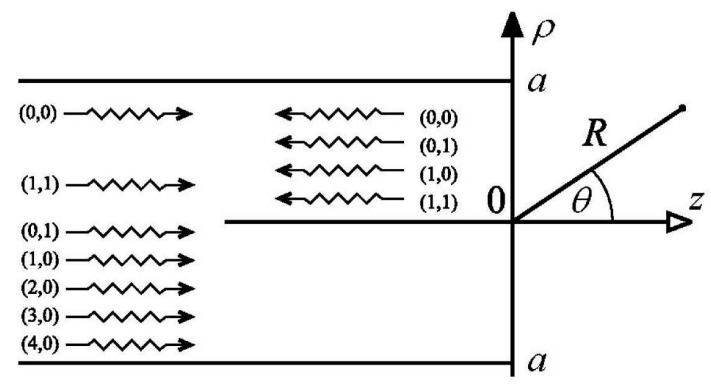

Fig. 1. Geometry of the problem.

For ducts important from practical point of view, as they constitute components of air-conditioning, ventilation or heating systems, jet engine casings etc., the incident wave is most frequently a superposition of a number of cut-on (propagating) modes i.e. modes cut-off frequency of which extends the excitation frequency. Then, the acoustic potential of the incident wave is a sum of single mode potentials and the resultant field can be expressed as a sum of the incident and diffracted field of the propagating modes $[6]$. In cylindrical coordinates system $(\varrho, \varphi, z)$ (Fig. 1) it reads

$$
\begin{aligned}
& \Phi(\varrho, \varphi, z, t)=\sum_{m, l} \Phi_{m, l}^{\mathrm{inc}}(\varrho, \varphi, z, t) \\
& +\sum_{m, l} \Phi_{m, l}^{\mathrm{dif}}(\varrho, \varphi, z, t) .
\end{aligned}
$$

For the single $(m, l)$ incident mode of amplitude $A_{m l}$ the resultant time-independent part of the potential takes the form [6]:

$$
\begin{gathered}
\Phi_{m l}(\varrho, \varphi, z)=A_{m l} \mathrm{e}^{\mathrm{i} m \varphi}\left[\frac{J_{m}\left(\mu_{m l} \frac{\varrho}{a}\right)}{J_{m}\left(\mu_{m l}\right)} \mathrm{e}^{\mathrm{i} \gamma_{m l} z}\right. \\
\left.+\sum_{n=1 / 0}^{\infty} R_{m l n} \frac{J_{m}\left(\mu_{m n} \frac{\varrho}{a}\right)}{J_{m}\left(\mu_{m n}\right)} \mathrm{e}^{-\mathrm{i} \gamma_{m n} z}\right],
\end{gathered}
$$

where the axial wave number $\gamma_{m n}$ equals

$$
\gamma_{m n}=\sqrt{k^{2}-\mu_{m n}^{2} / a^{2}},
$$

$J_{m}$ is the Bessel function of $m$-th order, $\mu_{m n} / a$ is the radial wave number adequate for the imposed boundary condition (hard wall) and $\mu_{m n}$ is the $n$-th root of the derivative $J_{m}^{\prime}, R_{m l n}$ is the wave amplitude reflection/transformation coefficient [6]. It can be seen that at the outlet each of the incident modes $(m, l)$ transforms, due to diffraction, into all cut-on modes of the same circumferential order, i.e. $(m, l) \rightarrow(m, n)$. The summation over subscript $n$ is carried out to infinity, even though for $\mu_{m n}>k a$, the respective factors represent waves of purely imaginary axial wave numbers, which means that these waves are attenuated exponentially with the distance from the outlet. As the impedance of the outlet is calculated for $z=0$, in exact formulae these terms of the infinite series should be also taken into account. However, in practice the sum is usually cut-off at $n=N_{m}$, where $\mu_{m N_{m}} / a$ denotes the index of "the highest of the propagating modes" (with the real axial wave number) of circumferential order $m$ at a given $k a$. Such a procedure was applied in numerical calculations presented below. The $n=N_{m}$ number can be as well derived from the relation $\mu_{m N_{m}+1} \geq k a>\mu_{m N_{m}}$.

\section{The acoustic specific impedance of the duct outlet}

To derive the acoustic impedance of the duct outlet one has to calculate the acoustic pressure $p=\rho_{0} \partial_{t} \Phi$, the acoustic velocity $\boldsymbol{v}=-\nabla \Phi$ and its normal component $v_{\boldsymbol{n}}$, the root mean square normal velocity $\left\langle\left|v_{\boldsymbol{n}}\right|^{2}\right\rangle=$ $\frac{1}{S} \int_{S} v_{\boldsymbol{n}} v_{\boldsymbol{n}}^{*} \mathrm{~d} s$, and the radiated power $\mathcal{P}=\int p v_{\boldsymbol{n}}^{*} \mathrm{~d} s$, because for the non-uniform pressure and velocity distribution on the duct cross-section the specific impedance is defined as [3]: 


$$
Z=\frac{\mathcal{P}}{S\left\langle v_{\boldsymbol{n}}^{2}\right\rangle}
$$

\subsection{Single mode excitation}

For given incident mode $(m, l)$, according to $(2),(4)$ and the formulae quoted in the text one obtains

$$
\begin{gathered}
Z_{m l}=\omega \rho_{0} \\
\times \frac{\sum_{n=1 / 0}^{\mathrm{N}_{\mathrm{m}}} \gamma_{m n} \mathcal{W}_{m n}\left(\delta_{n l}+2 \mathrm{i} \delta_{n l} \operatorname{Im} R_{m l l}-\left|R_{m l n}\right|^{2}\right)}{\sum_{n=1 / 0}^{\mathrm{N}_{\mathrm{m}}} \gamma_{m n}^{2} \mathcal{W}_{m n}\left(\delta_{n l}-2 \operatorname{Re} R_{m l l}+\left|R_{m l n}\right|^{2}\right)}, \\
\times \frac{X_{m l}=\operatorname{Re} Z_{m l}=\omega \rho_{0}}{\sum_{n=1 / 0}^{\mathrm{N}_{\mathrm{m}}} \gamma_{m n} \mathcal{W}_{m n}\left(\delta_{n l}-\left|R_{m l n}\right|^{2}\right)} \\
\times \frac{Y_{m l}^{\infty}=\operatorname{Im} Z_{m l}=-\omega \rho_{0} \mathcal{W}_{m n}\left(\delta_{n l}+2\left|R_{m l l}\right| \cos \theta_{m l l}+\left|R_{m l n}\right|^{2}\right)}{\sum_{n=1 / 0}^{\infty} \gamma_{m n}^{2} \mathcal{W}_{m n}\left(\delta_{n l}+2\left|R_{m l l}\right| \cos \theta_{m l l}+\left|R_{m l n}\right|^{2}\right)},
\end{gathered}
$$

where summation starts from $n=0$ only for $m=0$ (axisymmetrical excitation) and $\mathcal{W}_{m n}$ component appears as a result of integrating the product of the Bessel functions over the duct cross-section and is equal to $\left(1-m^{2} / \mu_{m n}^{2}\right)$, except for $m=0, n=0$, when it equals 1 [12].

\subsection{Multimode incident wave of the same or different circumferential orders $m$}

In the case of multimode excitation, when the incident wave is a superposition of many modes of the same circumferential order $m$ and so the acoustic potential $\Phi_{m}^{\text {inc }}(\varrho, \varphi, z)=\sum_{l} \Phi_{m l}^{\text {inc }}(\varrho, \varphi, z)$, the resulting acoustic field inside the duct is composed of terms representing incident and diffracted modes and takes the form

$\Phi_{m}(\varrho, \varphi, z)=\sum_{l} \Phi_{m l}(\varrho, \varphi, z)$, where $\Phi_{m l}$ was defined in Eq. (2). The acoustic pressure $p_{m}$ and velocity $\boldsymbol{v}_{m}$ have to be calculated from the potential $\Phi_{m}$ and the specific impedance of the outlet can be symbolically expressed as $Z_{m}=\frac{\mathcal{P}_{m}}{S\left\langle v_{m \boldsymbol{n}}^{2}\right\rangle}$, where the subscript $\boldsymbol{n}$ means, as before, the component of the acoustic velocity $\boldsymbol{v}_{m}$ normal to the duct outlet. To derive formulae for the specific acoustic impedance in multimode excitation, it is convenient to apply the matrix formalism, as the quadruple sums appear in calculations leading to the result.

Introducing for a given matrix $\boldsymbol{X}$ a symbol $(\boldsymbol{X})_{n}^{l}$ representing the element of line $l$ and column $n$ of this matrix, the specific acoustic impedance takes the form

$$
Z_{m}=\frac{\left(\boldsymbol{A}(\boldsymbol{I}+\boldsymbol{R}) \boldsymbol{M}^{(1)} \boldsymbol{A}^{\dagger}\right)_{1}^{1}}{S\left(\boldsymbol{A}(\boldsymbol{I}-\boldsymbol{R}) \boldsymbol{M}^{(2)} \boldsymbol{A}^{\dagger}\right)_{1}^{1}} \omega \rho_{0} .
$$

The matrix elements $\boldsymbol{A}, \boldsymbol{R}$ and $\boldsymbol{M}^{(k)}$ for $k \in\{1,2\}$ are defined as:

$$
\begin{aligned}
& A_{n}^{1} \equiv A_{m n}, \quad n=1, \ldots, N_{m}, \\
& R_{n}^{l} \equiv R_{m l n}, \quad l, n=1, \ldots, N_{m}, \\
& M_{n}^{(k) l} \equiv \frac{\left(\delta_{l n}-R_{l}^{n *}\right) \gamma_{m l}^{k} \mathcal{W}_{m l}}{J_{m}^{2}\left(\mu_{m l}\right)}, \quad l, n=1, \ldots, N_{m} .
\end{aligned}
$$

The explicit forms of these matrices are as follows:

$$
\begin{aligned}
\boldsymbol{A} & =\left(\begin{array}{cccc}
A_{m 1} & A_{m 2} & \ldots & A_{m N_{m}}
\end{array}\right), \\
\boldsymbol{R} & =\left(\begin{array}{cccc}
R_{m 11} & R_{m 12} & \ldots & R_{m 1 N_{m}} \\
R_{m 21} & R_{m 22} & \ldots & R_{m 2 N_{m}} \\
\vdots & \vdots & \ddots & \vdots \\
R_{m N_{m} 1} & R_{m N_{m} 2} & \ldots & R_{m N_{m} N_{m}}
\end{array}\right),
\end{aligned}
$$

$$
\boldsymbol{M}^{(k)}=\left(\begin{array}{cccc}
\frac{\left(1-R_{m 11}^{*}\right) \gamma_{m 1}^{k} \mathcal{W}_{m 1}}{J_{m}^{2}\left(\mu_{m 1}\right)} & \frac{-R_{m 21}^{*} \gamma_{m 1}^{k} \mathcal{W}_{m 1}}{J_{m}^{2}\left(\mu_{m 1}\right)} & \ldots & \frac{-R_{m N_{m} 1}^{*} \gamma_{m 1}^{k} \mathcal{W}_{m 1}}{J_{m}^{2}\left(\mu_{m 1}\right)} \\
\frac{-R_{m 12}^{*} \gamma_{m 2}^{k} \mathcal{W}_{m 2}}{J_{m}^{2}\left(\mu_{m 2}\right)} & \frac{\left(1-R_{m 22}^{*}\right) \gamma_{m 2}^{k} \mathcal{W}_{m 2}}{J_{m}^{2}\left(\mu_{m 2}\right)} & \ldots & \frac{-R_{m N_{m} 2}^{k} \gamma_{m 2} \mathcal{W}_{m 2}}{J_{m}^{2}\left(\mu_{m 2}\right)} \\
\vdots & \vdots & \ddots & \vdots \\
\frac{-R_{m 1 N_{m}}^{*} \gamma_{m N_{m}}^{k} \mathcal{W}_{m N_{m}}}{J_{m}^{2}\left(\mu_{m N_{m}}\right)} & \frac{-R_{m 2 N_{m}}^{*} \gamma_{m N_{m}}^{k} \mathcal{W}_{m N_{m}}}{J_{m}^{2}\left(\mu_{m N_{m}}\right)} & \ldots & \frac{\left(1-R_{m N_{m} N_{m}}^{*}\right) \gamma_{m N_{m}}^{k} \mathcal{W}_{m N_{m}}}{J_{m}^{2}\left(\mu_{m N_{m}}\right)}
\end{array}\right)
$$

The $\boldsymbol{I}$ symbol represents a unit matrix of size $N_{m} \times N_{m}$, while $\boldsymbol{A}^{\dagger}$ means Hermitian conjugate of the matrix $\boldsymbol{A}$ :

$$
\boldsymbol{A}^{\dagger}=\left(\begin{array}{c}
A_{m 1}^{*} \\
A_{m 2}^{*} \\
\vdots \\
A_{m N_{m}}^{*}
\end{array}\right) \text {. }
$$

The wave propagating inside the duct and heading the outlet represents, in its most general form, a superposition of modes of different radial $l$ and circumfer- ential $m$ order, therefore the acoustic potential of the incident wave reads $\Phi_{\text {inc }}(\varrho, \varphi, z)=\sum_{m} \Phi_{m}^{\text {inc }}(\varrho, \varphi, z)=$ $\sum_{m} \sum_{l} \Phi_{m l}^{\mathrm{inc}}(\varrho, \varphi, z)$. Excitation of that kind is frequently considered in papers related to noise propagating from the turbofan engine inlet (the outlet on the fan side). In general, the number of modes which are cut-on increases abruptly with the non-dimensional frequency (the Helmholtz number) $k a$, and so for $k a=10$ the total number of the cut-on modes is $N_{10}=18$, for $k a=20, N_{20}=59$, whereas for $k a=30$ it increases up to $N_{30}=126$. However, in numerous applications, 
because of specific features and symmetries of the sound sources or the devices located inside the duct [13, 14], the modes of selected circumferential order will be excited particularly strongly [15]. This takes place inside turbofan engines, with a defined number of compressor van and fan blades.

The specific impedance of the outlet can be symbolically expressed as

$$
Z=\frac{\sum_{m} \mathcal{P}_{m}}{S \sum_{m}\left\langle v_{m \boldsymbol{n}}^{2}\right\rangle},
$$

which means that it can be expressed as a fraction in which the numerator is a sum over $m$ of numerators of Eq. (5), and respectively - the denominator is a sum of dominators of $(5)$

\section{Numerical calculations}

Numerical calculations were carried on according to Eqs. (4)-(8) and (14) and also formulae inserted in the text. They include the single mode non-axial excitation, but also the multimode incident wave of a given or different circumferential order. The case of axisymmetric incident wave was analysed in earlier paper [3]. Figures 2-7 present the relative specific impedance $Z^{\mathrm{R}}$, defined as ratio of the specific impedance to the characteristic resistance of the medium $\rho_{0} c$, thus $Z^{\mathrm{R}}=Z / \rho_{0} c$.

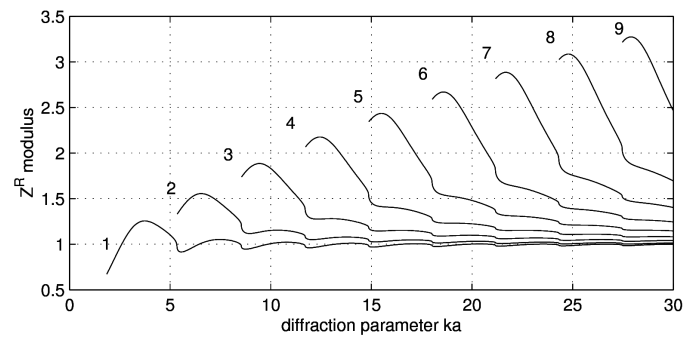

Fig. 2. Modulus of the relative specific acoustic impedance $Z^{\mathrm{R}}$ of the duct outlet for single asymmetric incident modes $(1, l)$ versus diffraction parameter $k a$. Numbers by the curves represent corresponding $l$ values.

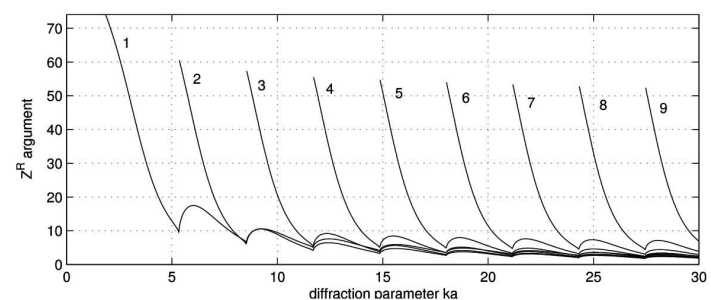

Fig. 3. Argument of the relative specific acoustic impedance $Z^{\mathrm{R}}$ of the duct outlet for single asymmetric incident modes $(1, l)$ versus diffraction parameter $k a$. Numbers by the curves represent corresponding $l$ values.

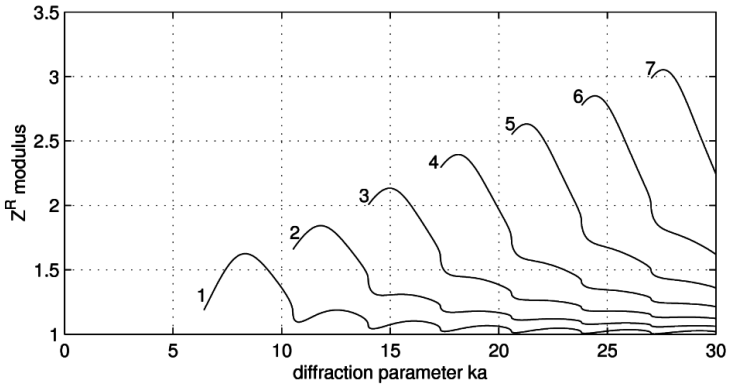

Fig. 4. The same as in Fig. 2, but for incident modes $(5, l)$.

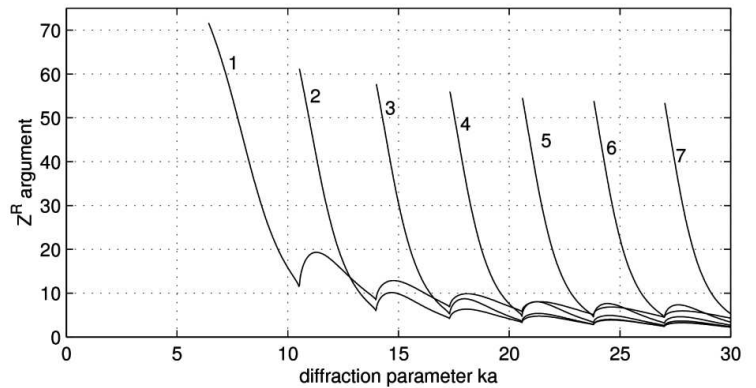

Fig. 5. The same as in Fig. 3, but for incident modes $(5, l)$.

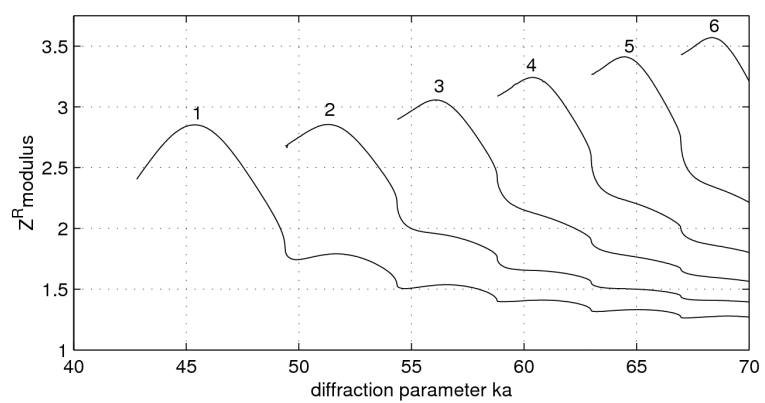

Fig. 6. The same as in Fig. 2, but for incident modes $(40, l)$.

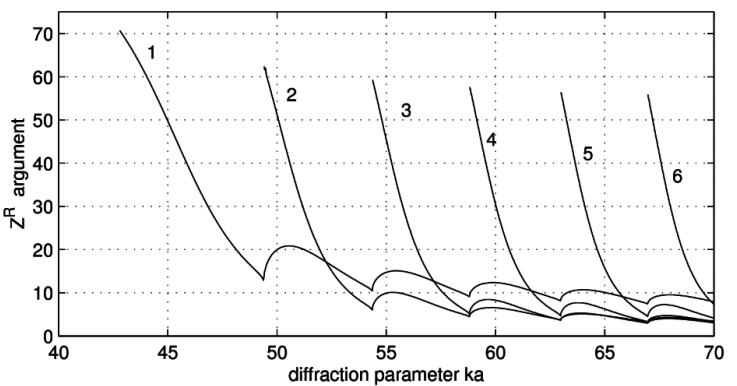

Fig. 7. The same as in Fig. 3, but for incident modes $(40, l)$. 


\subsection{Single non-axial mode $(m \neq 0)$ excitation}

The impedance of the single non-axial mode was computed and presented on graphs for the circumferential order $m=[1,5,40]$ and all possible values of radial order which represent propagating mode in the range of considered diffraction parameter $k a-[0-30]$ for $m=1$ and $m=5$ and $[40-70]$ for $m=40$, as the cut-on reduced frequency of the mode $(40,1)$ extends 40 [12]. The results confirm the modal character of the specific acoustic impedance of the outlet. For large values of $k a$ the real part tends to 1 , the imaginary part - to 0 , the minima appear for $k a$ equal to the reduced cut-on frequency of a subsequent mode with the maxima between them. These main features can be observed for any number of the mode circumferential order, same for $m=1$ or $m=40$.

\subsection{Multimode incident wave of a given circumferential order $m$}

As was mentioned before, inside jet engines, but also ventilation, heating or cooling systems etc., when the duct radii are large and the excitation frequencies are high, the wave heading to the outlet is usually superposition of radial and circumferential modes.

Multimode excitation complicates quantitative analysis of the resultant field, as it needs deriving complex amplitudes of consecutive modes. These amplitudes can be derived by solving inhomogeneous wave equation for a given source distribution inside the duct [13] or by adopting some additional assumptions. The inhomogeneous wave equation has been solved only for some simple inside-duct source distributions, which in turn do not model properly the features of real sources such as large fans mounted inside jet engines or ventilation/air-conditioning systems. The possible way to overcome this difficulty consists in assuming arbitrary amplitudes of modes or consider some specific features of the analysed setup, that would allow to predict which modes might have predominated in the incident wave or assume EEpM, as was proposed by Snakowska [9] and applied by other authors $[7,8]$. Mathematically the last assumption is legitimised by the orthogonality of the Bessel functions [12] appearing in the solution of the wave Eq. (2), and experimentally — by results obtained by Bolleter and Crocker [16].

Below, we present numerical results for the multimode incident wave, composed of all cut-on modes of a given circumferential order $m$ versus $k a$. The power of the incident wave has been assumed constant and distributed between the cut-on modes in equal parts. As a result, the imposed amplitudes moduli have been chosen according to the assumption on EEpM, while the phases have been assumed random. The numerical calculations were carried out by means of the Monte Carlo method, with $10^{4}$ realisations.

Assumption of EEpM in the multimode incident wave allows to determine the consecutive mode amplitudes [9]:

$$
\begin{aligned}
& \quad\left|A_{m n}\right|:\left|A_{m^{\prime} n^{\prime}}\right|=\sqrt{\frac{\gamma_{m^{\prime} n^{\prime}} \mathcal{W}_{m^{\prime} n^{\prime}}}{\gamma_{m n} \mathcal{W}_{m n}}}, \\
& \text { so } \\
& \qquad\left|A_{m n}\right|=\frac{1}{N_{\text {tot }}} \sqrt{\frac{2 \mathcal{P}^{\text {inc }}}{\pi a^{2} \rho_{0} c k \gamma_{m n} \mathcal{W}_{m n}}},
\end{aligned}
$$

where $N_{\text {tot }}$ denotes the number of modes present in the incident wave and $\mathcal{P}^{\text {inc }}$ - its power.

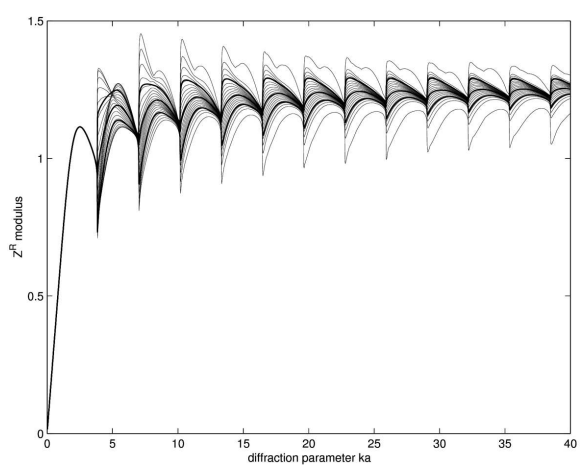

Fig. 8. Modulus of the relative specific acoustic impedance $Z^{\mathrm{R}}$ of the duct outlet for multimode incident wave of circumferential order $m=0$ versus diffraction parameter $k a$ with amplitudes selected according to EEpM assumption and random phases. Fine lines represent percentiles, bold lines - the expected value $E\left(Z^{\mathrm{R}}\right)$ and $E \pm \sigma$, where $\sigma$ means standard deviation.

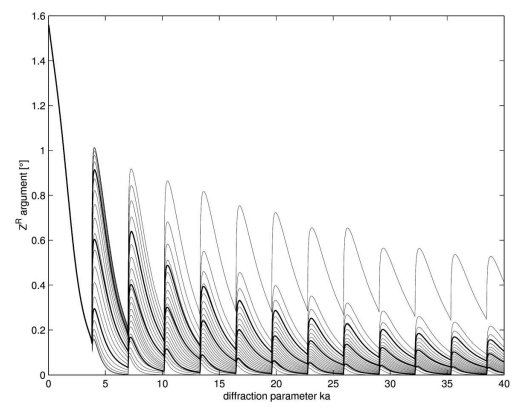

Fig. 9. Argument of the relative specific acoustic impedance $Z^{\mathrm{R}}$ of the duct outlet for multimode incident wave of circumferential order $m=0$ versus diffraction parameter $k a$ with amplitudes selected according to EEpM assumption and random phases. Fine lines represent percentiles, bold lines - the expected value $E\left(Z^{\mathrm{R}}\right)$ and $E \pm \sigma$, where $\sigma$ means standard deviation.

The results are presented in Figs. 8-14. Figures 8-13 present the modulus and argument of the relative impedance versus $k a$ for $m=[0,1,5]$. Each figure contains curves of the maximum and minimum values and percentiles drawn with the step $5 \%$ (fine lines) as well as the expected value $E\left(Z^{\mathrm{R}}\right)$ and the expected value plus/ minus the standard deviation $\sigma\left(Z^{\mathrm{R}}\right)$ (bold lines). As before - in single mode excitation, the graphs reveal their modal character with modulus minima at cut-on reduced 


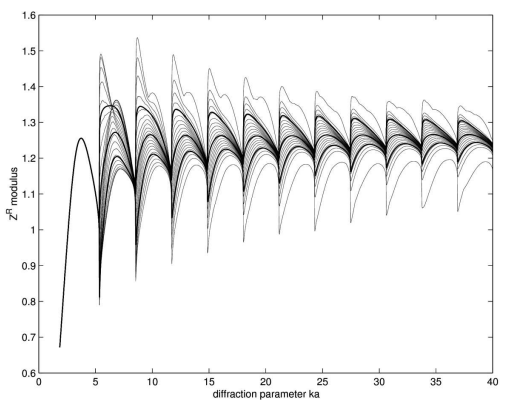

Fig. 10. The same as in Fig. 8, but for incident modes $m=1$.

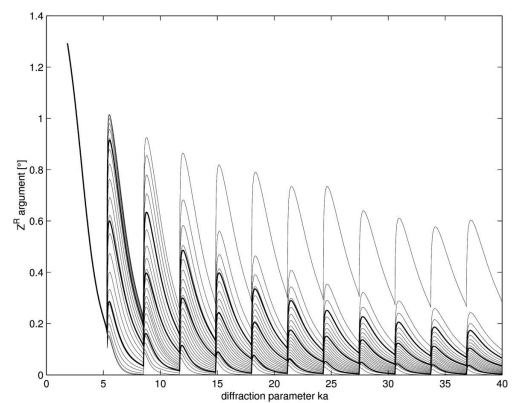

Fig. 11. The same as in Fig. 9, but for incident modes $m=1$.

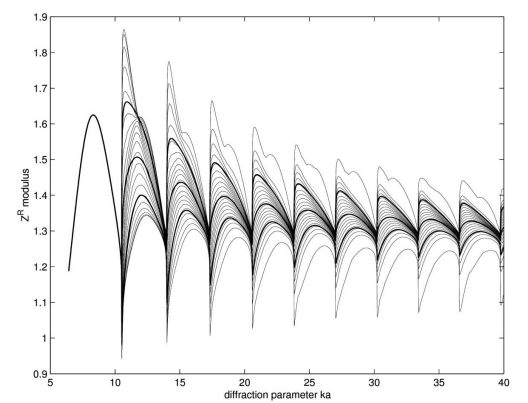

Fig. 12. The same as in Fig. 8, but for incident modes $m=5$.

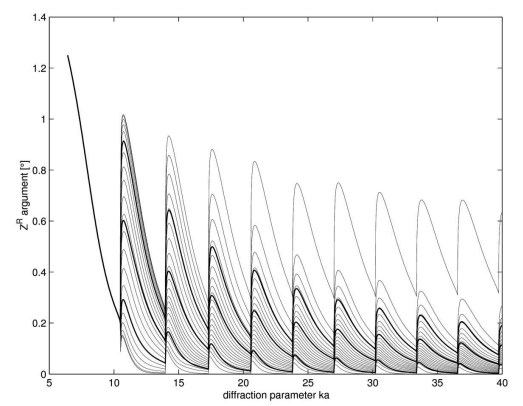

Fig. 13. The same as in Fig. 9, but for incident modes $m=5$.

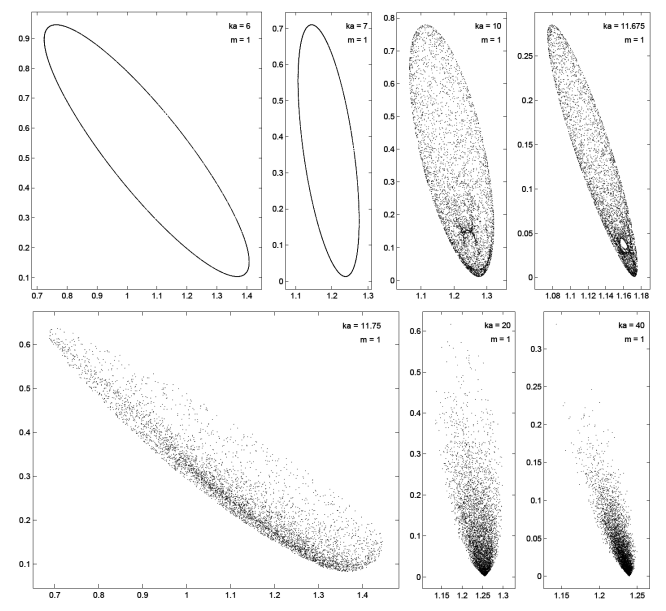

Fig. 14. Distribution of the real $\operatorname{Re} Z^{\mathrm{R}}$ (horizontal axis) and imaginary $\operatorname{Im} Z^{\mathrm{R}}$ (vertical axes) parts of the relative impedance for the circumferential order $m=1$ and different values of $k a$.

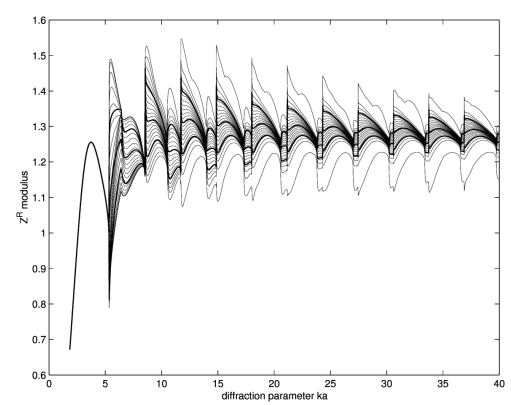

Fig. 15. The same as in Fig. 8, but for circumferential orders $m=[1,5]$.

frequencies of consecutive modes. Limiting the range of obtained results to [5-95] percentiles significantly narrows the range of the impedance values, especially the argument (cf. Figs. 9, 11, 13). Figure 14 presents distribution of the real $\operatorname{Re} Z^{\mathrm{R}}$ and imaginary part $\operatorname{Im} Z^{\mathrm{R}}$ of the relative impedance for the circumferential order $m=1$ and different values of $k a$. If $k a=6$, only two

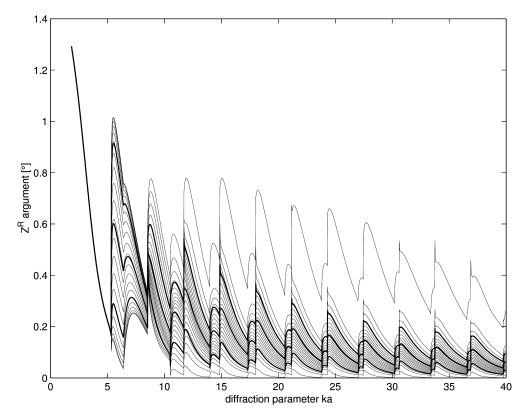

Fig. 16. The same as in Fig. 9, but for circumferential orders $m=[1,5]$. 
modes constitute the incident wave and the distribution of the impedance values takes the form of an elipse axis of which rotates with increasing $k a$ as can be seen on the graph for $k a=7$, up to the appearance of the third mode in the incident wave (cf. $k a=10$ ). The next two graphs (Figs. 15 and 16) present the distribution for $k a$ slightly below $k a=11.675$ and above $k a=11.75$ the cut-on frequency of the fourth mode and it can be observed how it changes the space of possible values of the real $\operatorname{Re} Z^{\mathrm{R}}$ and imaginary part $\operatorname{Im} Z^{\mathrm{R}}$.

\subsection{Multimode incident wave of different circumferential orders}

Propagation of wave composed of modes of different circumferential orders was discussed by Joseph et al. [15] for the noise raised inside a turbofan engine. In the presented numerical simulation excitation of the acoustic wave, it was assumed to be due to periodic wake interaction at the blade passing frequency by the stator vanes from the rotor stage. The duct, stator and fan parameters were standardised according to modern turbofan engines. The rotor was assumed to comprise of $B=25$ blades, the stator stage $-V=30$. The orders of circumferential propagating modes were derived from the criterion formulated by Tyler and Sofrin [17]:

$$
m=n B-p V, \quad p= \pm 1, \pm 2, \pm 3,
$$

which, together with the cut-on condition accounting for the blade tip Mach number and the intake flow Mach number [8], indicate that only the $m=5$ mode can propagate, with other modes being cut-off. Therefore computer simulations were carried out for $m=5$ modes, excited through rotor-stator interaction and additionally $m=1$ propagating modes. Below, the relative acoustic impedance, for a sum of propagating modes of these two circumferential orders are presented in Figs. 15 and 16. Numerical study was performed under the discussed assumptions on EEpM and random phases.

\section{Conclusions}

The formulae for the specific acoustic impedance of the outlet of a hard semi-infinite cylindrical waveguide for multimode incident wave propagating towards the open end have been derived accounting for diffraction phenomena, resulting not only in incident modes reflection but also their transformation in all possible modes of the same circumferential order. The assumption of multimode incident wave has brought the theoretical model closer to what is being observed in practice, especially when ducts of large radius, such as heating or air conditioning systems or turbojet engines, are considered. The exact mathematical formulae containing infinite series have been adopted to numerical calculations by neglecting in the diffracted term of the acoustic potential the attenuated modes, with pure imaginary axial wave number. Numerical results presented on graphs have been obtained based on assumption of EEpM in the incident wave and of random mode phases. The assumption of random phase was realised by means of the Monte Carlo method. The results of numerical calculations carried out according to derived theoretical formulae for single and multi-mode incident wave were presented on graphs. The detailed analysis of many graphs, some of which were included in this paper, indicate strong dependence of the outlet impedance on the modes phases and also confirm significant difference between the approach presented in the paper and the plane wave approximation, frequently applied in technical considerations. The above-presented results may serve in future as a tool for deriving analogous formulae for the impedance of a soft or sound absorbing wall duct outlet.

\section{References}

[1] Rayleigh, Lord, The Theory of Sound, Dover, New York 1945 (reprinted).

[2] E. Zorumski, J. Acoust. Soc. Am. 54, 1667 (1973).

[3] A. Snakowska, R. Wyrzykowski, Arch. Acoust. 13, 137 (1988).

[4] H. Levine, J. Schwinger, Phys. Rev. 73, 383 (1948).

[5] L.A. Weinstein, The Theory of Diffraction and the Factorization Method (Generalised Wiener-Hopf Technique), Golem Press, Boulder, Colorado 1969.

[6] A. Snakowska, R. Wyrzykowski, Arch. Acoust. 11, 261 (1986).

[7] G. Gabard, R.J. Astley, J. Sound Vib. 315, 110324 (2008).

[8] P. Joseph, C.L. Morfey, J. Acoust. Soc. Am. 105, 2590 (1999).

[9] A. Snakowska, Acustica 79, 155 (1993).

[10] E. Skudrzyk, The Foundations of Acoustics, Springer-Verlag, Wien 1971.

[11] B. Noble,Methods Based on the Wiener-Hopf Technique for the Solution of Partial Differential Equations, Pergamon Press, London 1958.

[12] G.N. Watson, A Treatise on the Theory of Bessel Functions, Cambridge University Press, Cambridge 1958.

[13] G.W. Johnston, K. Ogimoto, J. Acoust. Soc. Am. 68, 1858 (1980).

[14] G.W. Johnston, K. Ogimoto, J. Acoust. Soc. Am. 68, 1871 (1980).

[15] P. Joseph, P.A. Nelson, M.A. Fisher, J. Acoust. Soc. Am. 106, 766 (1999).

[16] U. Bolleter, M. Crocker, J. Acoust. Soc. Am. 51, 1439 (1972).

[17] J.M. Tyler, T.G. Sofrin, Trans. Soc. Automot. Eng. 70,309 (1962). 